\title{
Soforttypallergie: H1-Blocker ungeschlagen
}

\section{Auch wenn mittlerweile eine fast unüberschaubare Zahl an Boten- stoffen der allergischen Reaktion bekannt ist, behält doch die Antagonisierung der Histaminwirkung seine herausragende Rolle in der symptomatischen Behandlung von Typ-I-Allergien. Und das Potential der Anthistaminika scheint noch längst nicht ausgereizt.}

Z u einem vernünftigen Antihistaminika-Einsatz in der Praxis gehört es, daß man weiß, was man nicht davon erwarten darf, erklärte kürzlich B. Wüthrich (Zürich): Da das Histamin zwar ein sehr wichtiger, aber keineswegs der einzige Mediator im Faktorengeflecht der allergischen Entzündung ist, wird ein Patient mit einem Antihistaminikum allein nie ganz beschwerdefrei; vor allem aber wird die Entzündung nicht unterdrückt, die sich ja später im Ablauf der Reaktionskaskade entwickelt.

Weiterhin muß man beachten, erinnerte Wüthrich, daß das Gewebshormon, sobald sich Symptome manifestieren, bereits an den Rezeptor in den Gefäßen etc. gebunden ist, mit anderen Worten: Antihistaminika sind Prophylaktika, d.h., sie müssen verabreicht werden, bevor der Patient mit dem Allergen in Berührung kommt.

\section{Praktische Empfehlungen für Urtikaria-Patienten}

So gilt beispielsweise für den Urtikaria-Patient: Er kann mit einem H1Blocker nie gänzlich von seinen Symptomen befreit werden. Allerdings wird er unter der Medikation kaum mehr Juckreiz verspüren, und die Schwellungen gehen zurück. Nur das Erythem bleibt; darüber muß man den Patienten vor Beginn der Behandlung informieren, um von vornherein Enttäuschungen vorzubeugen.

Für die Praxis empfahl Wüthrich bei der chronisch idiopathischen Urtikaria eine Mehrstufen-Kombinationstherapie wie die in Zürich prakti-

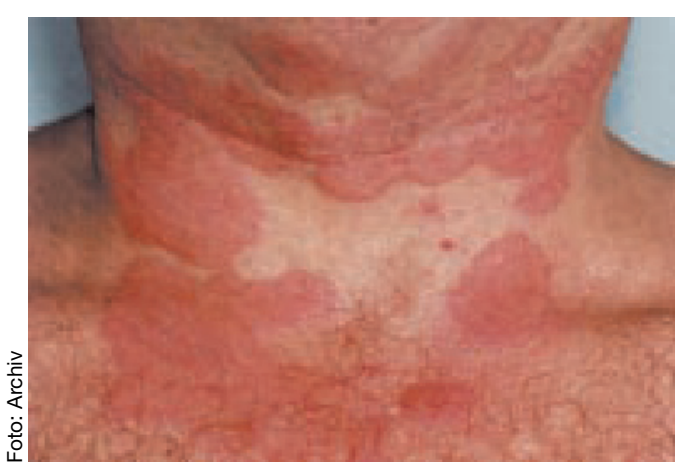

Flüchtige Quaddeln an Hals und Brust - ein typisches Bild bei Urtikaria. Antihistaminika lindern Juckreiz und Schwellung, das Erythem geht aber nicht vollständig zurück.

zierte „3-Z-Therapie“: Zunächst verschreiben die Schweizer Kollegen Cetirizin $\left(\right.$ Zyrtec $\left.^{\circledR}\right)$; wenn diese Medikation allein nicht hilft, zusätzlich einen H2-Antagonisten wie Ranitidin (z.B. Zantic $^{\circledR}$ ), und - falls erforderlich wird das Schema noch durch die abendliche Gabe von Ketotifen (z.B. Zaditen ${ }^{\circledR}$ ) als Mastzellprotektor ergänzt. Wichtig: Bei Urtikaria eher die höhere Dosis verwenden.

\section{Neue Antihistaminika - mehr als selektive H1-Antagonisten}

Alle modernen Antihistaminika wie beispielsweise Cetirizin, Loratadin, Fexofenadin und Mizolastin zeigen antiallergische Eigenschaften. Schon vor mehreren Jahren hat man gesehen, daß Cetirizin den im Rahmen der allergischen Spätphase stattfindenden Einstrom der eosinophilen Granulozyten ins Gewebe hemmt. Heute weiß man, daß die Hemmung darauf beruht, daß die Antihistaminika der neueren Generation die Expression von interzellulären Adhäsionsmolekülen (z.B. ICAM-1) unterdrückt, wie man auch in Proben der Nasenschleimhaut von Allergikern zeigen konnte. Da die Entzündungszellen, um aus der Blutbahn ins Gewebe zu gelangen, zunächst an diese Rezeptoren binden müssen, wird durch die Hemmung deren Expression auch die Rekrutierung der Eosinophilen supprimiert.

Den Adhäsionsmolekülen kommt überhaupt eine offenbar wichtige Rolle bei der persistierenden allergischen Entzündung zu: Man weiß heute, daß auch nach Abklingen der klinischen Symptome sowie der Eosinophilen-Aktivierung wenn die Allergenexposition längst vorüber ist - die erhöhte Dichte von ICAM-1-Molekülen auf den Gefäß-Endothelzellen bestehen bleibt - zum Teil über Wochen. So lassen sich auch die Resultate einer interessanten Arbeit aus Genua erklären, in welcher die regelmäßige Einnahme von Cetirizin der bedarfsorientierten Einnahme überlegen war.

Rhinoviren den Weg versperrt

Da gerade auch Rhinoviren die ICAM-1-Moleküle als Eintrittspforte benutzen, könnte eine Antihistaminika-Therapie womöglich auch die erhöhte Häufigkeit von Infekten des Respirationstrakts bei Allergikern reduzieren, spekulierte Wüthrich.

Rhinitis-Patienten und Kleinkinder mit atopischem Ekzem sind bekanntlich Kandidaten für ein Asthma bronchiale. Die seit mehreren Jahren laufende ETAC-Studie (Early Treatment of the Atopic Child) soll prüfen, ob die frühzeitige Intervention mit einem Antihistaminikum (Cetirizin) die gefürchtete Entwicklung in diese Richtung verhindern kann. „Wir sind alle gespannt auf die ersten Ergebnisse, die noch in diesem Jahr bekannt gegeben werden", so der Züricher Experte.

(wpa)

Quelle: Vortrag von Brunello Wüthrich (Zürich) auf der 16 Fortbildungswoche für praktische Dermatologie und Venerologie, München, 27.7.1998. 